\title{
EL FEMUR CORTO NO ES UN BUEN MARCADOR DE SINDROME DE DOWN EN FETOS PERUANOS
}

\author{
Oscar Antonio Limay Rios ${ }^{1}$, Frandy Macha Castañeda ${ }^{2}$, Jaime Ingar Pinedo ${ }^{3}$, Walter Castillo Urquiaga ${ }^{4}$, \\ Walter Ventura Laveriano ${ }^{4}$, Mario Zarate Girao ${ }^{4}$.
}

\begin{abstract}
RESUMEN
Objetivo. Determinar la similitud o diferencia de longitud del fémur de fetos normales comparados a los fetos con síndrome de Down en el Instituto Materno Perinatal periodo 2011-2015. Metodología. Es un estudio de investigación cuantitativo, retrospectivo, observacional, descriptivo Correlacional y Transversal. Nuestra población fue todos los fetos del Instituto Nacional Materno Perinatal en el periodo 2011-2015, que tuvieron la medida de Longitud del Fémur y la base de datos completa, que cumplen con los criterios de Inclusión y Exclusión. Resultados. En la curva normal de longitud de fémur para fetos sanos se encontró, percentil 95 estuvo por encima de los demás percentiles, con un valor máximo que oscila entre 93,09 mm y un valor mínimo que varía entre $16,34 \mathrm{~mm}$, En la curva de longitud de fémur para fetos con síndrome de Down se encontró que la curva del percentil 95 estuvo por encima de los demás percentiles, con un valor máximo que oscila entre $90,48 \mathrm{~mm}$ y un valor mínimo que varía entre $14,23 \mathrm{~mm}$, en la comparación de las curvas de la longitud de fémur de los fetos sanos con respecto a los fetos con síndrome de Down, los valores de longitud del fémur son diferentes, observándose que la curva de los fetos con síndrome de Down tiene una tendencia a valores bajos entre $8,78 \mathrm{~mm}$ el mínimo y el máximo con $56,43 \mathrm{~mm}$. Conclusión. Al comparar ambas curvas de longitud del fémur lo más representativo fue que los fetos con síndrome de Down tienen longitudes de fémur muchos menores comparados a los fetos normales.
\end{abstract}

Palabra clave: Longitud del fémur; Síndrome de Down; Fémur corto (Fuente: DeCS BIREME).

\section{SHORT FEMUR IS NOT A GOOD MARKER OF DOWN SYNDROME IN PERUVIAN FETUS}

\begin{abstract}
Objective. To determine the similarity or difference in femur length of normal fetuses compared to fetuses with Down syndrome at the Maternal Perinatal Institute period 2011-2015. Methodology. It is a quantitative, retrospective, observational, descriptive and cross-sectional descriptive study. Our population was all fetuses of the National Perinatal Maternal Institute in the period 2011-2015, which had the measure of Femur Length and the complete database, which meet the criteria of Inclusion and Exclusion. Result. In the normal curve of femur length for healthy fetuses was found, percentile 95 was above the other percentiles, with a maximum value ranging from $93,09 \mathrm{~mm}$ and a minimum value ranging between $16,34 \mathrm{~mm}$, in the femur length curve For fetuses with Down syndrome, the 95th percentile curve was found to be above the other percentiles, with a maximum value ranging from $90,48 \mathrm{~mm}$ to a minimum value ranging from $14,23 \mathrm{~mm}$, when comparing the length curves Of fetuses of healthy fetuses with respect to fetuses with Down syndrome, the values of femur length are different, observing that the curve of the fetuses with Down syndrome has a tendency to low values between $8,78 \mathrm{~mm}$ the minimum and the maximum With $56,43 \mathrm{~mm}$. Conclusion. When comparing both curves of femur length, the most representative was that fetuses with Down syndrome have many lower femur lengths compared to normal fetuses.
\end{abstract}

Key word: Length of the femur; Down syndrome; Short femur(Source: MeSH NLM).

\section{INTRODUCCIÓN}

El impacto que tienen los defectos congénitos sobre la salud de las personas afectadas, sus familias y sobre la sociedad en su conjunto es muy considerable. En España se estima que existe una prevalencia de anomalías cromosómicas hasta 2010 de $1,49 \%$ de los recién nacidos vivos ${ }^{1}$.

En Catalunya la tasa del síndrome de Down ha pasado de un 0,9 por mil en el 1993 a un 1,8 por mil en el año 2006 . Según la oficina de estadísticas e informática dellnstituto Nacional Materno Perinatal hubo un incremento en la tasa del síndrome de Down de un $1,2 \%$ en el 2013 a 1,3\% en el $2014^{2,3}$.
La importante morbilidad asociada en los individuos con síndrome de Down se acompaña de un alto coste económico, estimándose en 329750,63 euros por cada nuevo caso, constituyendo un cargo a lo largo de la vida de 1316 millones de euros ${ }^{4}$.

La estatura baja se ha reconocido como un rasgo de los individuos con síndrome de Down, asociado a huesos largos proximales desproporcionadamente cortos (fémur) ${ }^{5}$.

Numerosos estudios han documentado que una longitud relativamente corto del fémur fetal en el segundo trimestre de la gestación plantea la sospecha de aneuploidía. Un

\footnotetext{
Médico Gineco-Obstetra Asistente del Servicio de Medicina Fetal. Jefe de Unidad de Investigación del Instituto Nacional Materno Perinatal. Lima-Perú Estudiante de Facultad de Medicina Humana de la Universidad San Juan Bautista.Lima-Perú

Médico Gineco-Obstetra Jefe del Servicio de Medicina Fetal del Instituto Nacional Materno Perinatal. Lima-Perú.

4 Médico Gineco-Obstetra Asistente del Servicio de Medicina Fetal del Instituto Nacional Materno Perinatal. Lima-Perú
} 
fémur corto puede tener una sensibilidad del $24 \%$ con una tasa de falsos positivos del $4,7 \%$ en la identificación de Down síndrome ${ }^{6}$.

Este hecho hace necesaria la puesta en marcha de un correcto cribado en estos fetos con alto riesgo de morbimortalidad y en donde la medición de la longitud del fémur y otros marcadores ecográficos en el segundo trimestre pueden contribuir a una detección oportuna y un diagnóstico precoz ya que tienen una tasa de detección que oscila un 62 al $93 \%$, con una tasa de falsos positivos de $17 \%$ al $4.8 \%{ }^{7}$.

El fémur, sería anormal si está por debajo del percentil 5 o aplicando la regresión o fórmula: fémur esperado $=$ $9.645+0.9338 \times$ DBP. Se considera fémur corto cuando la relación fémur observado / fémur esperado da un valor de 0,91 . Este hallazgo identifica únicamente el $40 \%$ de los fetos con T-21 y falsos positivos del $5 \% 18$, pero si existen fémur corto y pliegue nucal > de $6 \mathrm{~mm}$, la detección se aumenta al $75 \%$ y con un $2 \%$ de falsos positivos 19 . La Razón de Verisimilitud para el fémur corto es de $1,6^{12}$.

El hallazgo ecográfico de fémur corto (longitud menor del percentil 5) en la valoración ecográfica rutinaria del feto del segundo trimestre plantea un desafío diagnóstico y de manejo. Clásicamente ha sido asociado a cromosomopatías y displasia esquelética.

Numerosos estudios han documentado que un fémur fetal relativamente corto en el segundo trimestre plantea la sospecha de aneuploidía. Un fémur corto puede tener una sensibilidad del $24 \%$ con una tasa de falsos positivos del $4,7 \%$ en la identificación de síndrome Down ${ }^{6}$.

Por tal motivo es importante comparar y saber si la longitud del fémur de los fetos peruanos sanos es igual o no al de los fetos con síndrome de Down para saber si aplica como predictor de síndrome de Down en nuestro medio.

\section{MATERIALES Y MÉTODOS}

El presente estudio es una investigación cuantitativo, retrospectivo, observacional, transversal, descriptivo Correlacional en el servicio de Medicina Fetal del Instituto Nacional Materno Perinatal (INMP). La población de estudio estuvo conformada por 3480 fetos sanos y 143 fetos con síndrome de Down del Instituto Nacional Materno Perinatal en el periodo 2011-2015, que tuvieron la medida de Longitud del Fémur y la base de datos completa, que cumplen con los criterios de Inclusión y Exclusión.

Este estudio fue aprobado por el Comité de Ética del Instituto Nacional Materno Perinatal.

La técnica de recolección de datos que se realizó en este trabajo de investigación es la observación y el instrumento son los registros donde se encuentran los datos (base de datos) que tiene el servicio de medicina fetal del Instituto Nacional Materno Perinatal.

Se procedió a la confección de una base de datos, conformada por una matriz estructurada en el programa Excel y se exporto la base de datos al programa SPSS versión 22 para su análisis, posteriormente se realizó la transformación natural logarítmica con regresión por estimación curvilínea de modelo inverso, y en donde se obtuvo los valores de los percentiles tanto 5, 50 y 95 . Posteriormente los datos fueron importados al Excel para la confección de las tablas, y estimación de las curvas.

\section{RESULTADOS}

Tabla 1. Percentiles normales de longitud del femur para fetos sanos del instituto nacional materno perinatal 20112015

\begin{tabular}{cccc}
\hline EG & P5 LF & P50 LF & P95 LF \\
\hline 14 & 11.03 & 13.42 & 16.34 \\
15 & 13.18 & 16.04 & 19.53 \\
16 & 15.41 & 18.75 & 22.83 \\
17 & 17.68 & 21.52 & 26.20 \\
18 & 19.99 & 24.33 & 29.61 \\
19 & 22.30 & 27.15 & 33.04 \\
20 & 24.62 & 29.96 & 36.47 \\
21 & 26.91 & 32.76 & 39.87 \\
22 & 29.19 & 35.53 & 43.24 \\
23 & 31.43 & 38.26 & 46.57 \\
24 & 33.64 & 40.94 & 49.84 \\
\hline 25 & 35.81 & 43.58 & 53.05 \\
26 & 37.93 & 46.17 & 56.20 \\
27 & 40.01 & 48.70 & 59.28 \\
\hline 28 & 42.04 & 51.18 & 62.30 \\
29 & 44.03 & 53.60 & 65.24 \\
30 & 45.97 & 55.95 & 68.11 \\
\hline 31 & 47.86 & 58.26 & 70.91 \\
32 & 49.70 & 60.50 & 73.64 \\
\hline 33 & 51.50 & 62.68 & 76.30 \\
\hline 34 & 53.24 & 64.81 & 78.90 \\
35 & 54.95 & 66.89 & 81.42 \\
\hline 36 & 56.61 & 68.91 & 83.88 \\
\hline 37 & 58.22 & 70.87 & 86.27 \\
38 & 59.79 & 72.79 & 88.61 \\
\hline 39 & 61.32 & 74.65 & 90.87 \\
40 & 62.82 & 76.47 & 93.09 \\
\hline
\end{tabular}

Fuente: Base de datos del servicio de medicina fetal del Instituto Nacional Materno Perinatal. 2011-2015.

Interpretación. Se observó una tasa de crecimiento de longitud de fémur en el percentil 5 de 1,5 a 2,32mm por semana gestacional, siendo el de mayor aumento entre las 19 a 20 semanas con $2,32 \mathrm{~mm}$ y el de menor aumento entre las $39-40$ semanas con $1,5 \mathrm{~mm}$. Asimismo se vislumbró una tasa de crecimiento de longitud de fémur en el percentil 50 de 1,82 a 2,82mm por semana gestacional, siendo el de mayor aumento entre las 1819 semanas con $2,82 \mathrm{~mm}$ y el menor aumento entre las 
34-40 semanas de gestación con 1,82mm. En cuanto al percentil 95 la velocidad de crecimiento de longitud del fémur fluctúo entre 2,22 a $3,43 \mathrm{~mm}$, siendo el de menor aumento entre las 39-40 semanas con 2,22 y el de mayor aumento las $19-20$ semanas con 3,43mm. Se encontró además para los fetos sanos valores de longitud de fémur desde las 14 a 40 semanas de gestación, observándose que para el percentil 5 a las 14 semanas se encontró un valor de $11,03 \mathrm{~mm}$, a las 28 semanas se encontró un valor de $42,04 \mathrm{~mm}$, a las 37 semanas $58.22 \mathrm{~mm}$ y a las 40 semanas $62,82 \mathrm{~mm}$. Asimismo para el percentil 50 a las 14 semanas se encontró un valor de 13,42, a las 28 semanas se encontró un valor de $51,18 \mathrm{~mm}$, a las 37 semanas $70,87 \mathrm{~mm}$ y a las 40 semanas 76,47 . De igual manera se observó que para el percentil 95 a las 14 semanas se encontró un valor de $16,34 \mathrm{~mm}$, a las 28 semanas se encontró un valor de 62,30 , a las 37 semanas $86,27 \mathrm{~mm}$ y a las 40 semanas $93,09 \mathrm{~mm}$.

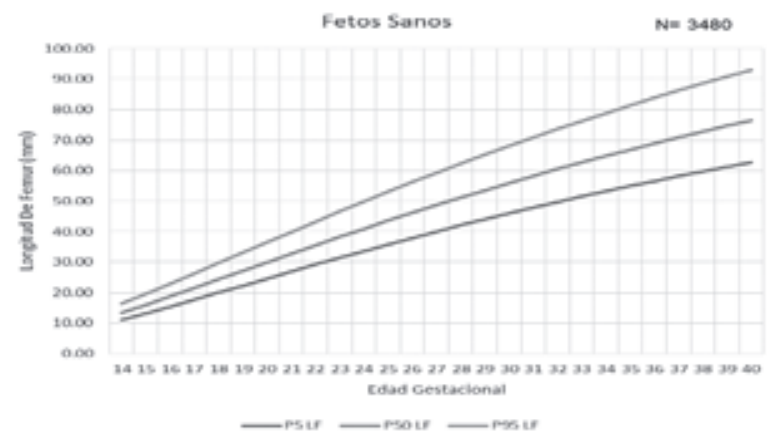

Gráfico 1. Curva normal de longitud del femur para fetos sanos del Instituto Nacional Materno Perinatal 2011-2015

Fuente: Base de datos del servicio de medicina fetal del Instituto Nacional Materno Perinatal 2011-2015.

Interpretación. En una población de 3480 fetos sanos del Instituto Nacional Materno Perinatal entre 2011-2015. Se observó una curva de crecimiento ascendente según edad gestacional ubicando al percentil 95 por encima de los demás percentiles, con un valor máximo que oscila entre $93,09 \mathrm{~mm}$ y un valor mínimo que varía entre 16,34 $\mathrm{mm}$, el percentil 50 oscila entre 13,42 a $76,47 \mathrm{~mm}$ y el percentil 5 oscila entre 11,03 a $62,82 \mathrm{~mm}$.

Interpretación. Se observó una tasa de crecimiento de longitud de fémur en el percentil 5 de 0,83 a $2.72 \mathrm{~mm}$ por semana gestacional, siendo el de mayor aumento de velocidad de crecimiento entre las 26 a 27 semanas con $2,72 \mathrm{~mm}$ y el menor aumento de velocidad de crecimiento entre las $32-33$ semanas con $0,83 \mathrm{~mm}$. Igualmente se vislumbró una tasa de crecimiento de longitud de fémur en el percentil 50 de 1,05 a 3,47mm por semana gestacional, teniendo como mayor aumento de velocidad de crecimiento entre las semanas 26-27 de gestación con $3,47 \mathrm{~mm}$ y el menor aumento entre las $17-38$ semanas de gestación con 1,05 mm. En cuanto al percentil 95 la velocidad de crecimiento de longitud del fémur fluctúa entre $1,33 \mathrm{~mm}$ y $4,22 \mathrm{~mm}$ siendo menor entre las $37-38$
Tabla 2. Percentiles de longitud del femur para fetos con Sindrome de Down del Instituto Nacional Materno Perinatal 2011-2015

\begin{tabular}{cccc}
\hline EG & P5 & P50 & P95 \\
\hline 14 & 8.78 & 11.18 & 14.23 \\
15 & 10.64 & 13.52 & 17.19 \\
16 & 13.29 & 16.87 & 21.41 \\
17 & 14.58 & 18.50 & 23.47 \\
18 & 16.63 & 21.08 & 26.73 \\
\hline 19 & 18.70 & 23.69 & 30.02 \\
\hline 20 & 20.78 & 26.32 & 33.34 \\
\hline 21 & 22.97 & 29.09 & 36.84 \\
\hline 22 & 25.05 & 31.71 & 40.15 \\
\hline 23 & 27.13 & 34.34 & 43.46 \\
24 & 29.14 & 36.88 & 46.68 \\
\hline 25 & 31.01 & 39.24 & 49.67 \\
26 & 32.98 & 41.73 & 52.81 \\
\hline 27 & 34.91 & 44.18 & 55.90 \\
28 & 37.63 & 47.65 & 60.10 \\
\hline 29 & 38.66 & 48.92 & 61.91 \\
\hline 30 & 40.48 & 51.22 & 64.82 \\
\hline 31 & 42.87 & 54.25 & 68.66 \\
\hline 32 & 43.99 & 55.67 & 70.45 \\
\hline 33 & 46.50 & 58.85 & 74.49 \\
\hline 34 & 47.33 & 59.91 & 75.83 \\
\hline 35 & 49.52 & 62.68 & 79.35 \\
\hline 36 & 51.18 & 64.79 & 82.02 \\
\hline 37 & 52.70 & 66.72 & 84.46 \\
\hline 38 & 54.18 & 68.60 & 86.85 \\
\hline 39 & 55.01 & 69.65 & 88.18 \\
40 & 56.43 & 71.46 & 90.48 \\
\hline
\end{tabular}

Fuente: Base de datos del servicio de medicina fetal del Instituto Nacional Materno Perinatal. 2011-2015.

semanas con $1.33 \mathrm{~mm}$ y mayor entre las $14-15$ semanas de gestación con 4,22 mm. Se encontró además en los fetos con síndrome de Down valores de longitud de fémur desde las 14 a 40 semanas de gestación, observándose que para el percentil 5 a las 14 semanas se encontró un valor de $8,78 \mathrm{~mm}$, a las 28 semanas se encontró un valor de $37,63 \mathrm{~mm}$, a las 37 semanas $52,70 \mathrm{~mm}$ y a las 40 semanas $56,43 \mathrm{~mm}$. Asimismo para el percentil 50 a

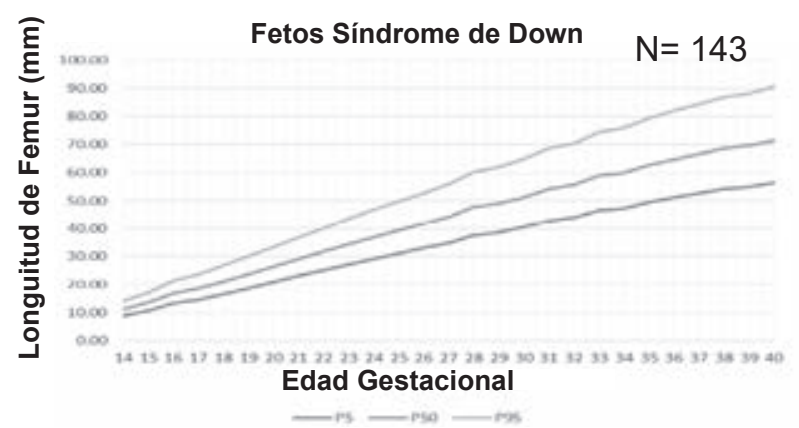

Gráfico 2. Curva de longitud del femur para fetos con Sindrome de Down del Instituto Nacional Materno Perinatal 2011-2015.

Fuente. Base de datos del servicio de medicina fetal del Instituto Nacional Materno Perinatal 2011-2015. 
las 14 semanas se encontró un valor de $11,18 \mathrm{~mm}$, a las 28 semanas se encontró un valor de $47,65 \mathrm{~mm}$, a las 37 semanas $66,72 \mathrm{~mm}$ y a las 40 semanas $90,48 \mathrm{~mm}$. De igual manera se observó que para el percentil 95 a las 14 semanas se encontró un valor de $14,23 \mathrm{~mm}$, a las 28 semanas se encontró un valor de $60,10 \mathrm{~mm}$, a las 37 semanas $84,46 \mathrm{~mm}$ y a las 40 semanas $90,48 \mathrm{~mm}$.

Interpretación. En una población de 143 fetos con síndrome de down del Instituto Nacional Materno Perinatal entre el 2011-2015. Se observó una curva de crecimiento ascendente según edad gestacional ubicando al percentil 95 por encima de los demás percentiles, cuyos valores máximos que oscilan entre $90,48 \mathrm{~mm}$ y un valor mínimo que varía entre $14,23 \mathrm{~mm}$, el percentil 50 oscila entre 11,18 a $71,46 \mathrm{~mm}$ y el percentil 5 oscila entre 8,78 a $56,43 \mathrm{~mm}$.

Tabla 3. Comparación de los percentiles de longitud de femur de los fetos sanos con los fetos Sindrome de Down del Instituto Nacional Materno Perinatal 2011-2015

\begin{tabular}{|ccccccc}
\hline EG & P5 LF & P50 LF P95 LF P5DOWN & P50DOWN & P95DOWN \\
\hline 14 & 11.03 & 13.42 & 16.34 & 8.78 & 11.18 & 14.23 \\
\hline 15 & 13.18 & 16.04 & 19.53 & 10.64 & 13.52 & 17.19 \\
\hline 16 & 15.41 & 18.75 & 22.83 & 13.29 & 16.87 & 21.41 \\
\hline 17 & 17.68 & 21.52 & 26.20 & 14.58 & 18.50 & 23.47 \\
\hline 18 & 19.99 & 24.33 & 29.61 & 16.63 & 21.08 & 26.73 \\
\hline 19 & 22.30 & 27.15 & 33.04 & 18.70 & 23.69 & 30.02 \\
\hline 20 & 24.62 & 29.96 & 36.47 & 20.78 & 26.32 & 33.34 \\
\hline 21 & 26.91 & 32.76 & 39.87 & 22.97 & 29.09 & 36.84 \\
\hline 22 & 29.19 & 35.53 & 43.24 & 25.05 & 31.71 & 40.15 \\
\hline 23 & 31.43 & 38.26 & 46.57 & 27.13 & 34.34 & 43.46 \\
\hline 24 & 33.64 & 40.94 & 49.84 & 29.14 & 36.88 & 46.68 \\
\hline 25 & 35.81 & 43.58 & 53.05 & 31.01 & 39.24 & 49.67 \\
\hline 26 & 37.93 & 46.17 & 56.20 & 32.98 & 41.73 & 52.81 \\
\hline 27 & 40.01 & 48.70 & 59.28 & 34.91 & 44.18 & 55.90 \\
\hline 28 & 42.04 & 51.18 & 62.30 & 37.73 & 47.75 & 60.43 \\
\hline 29 & 44.03 & 53.60 & 65.24 & 38.66 & 48.92 & 61.91 \\
\hline 30 & 45.97 & 55.95 & 68.11 & 40.48 & 51.22 & 64.82 \\
\hline 31 & 47.86 & 58.26 & 70.91 & 42.87 & 54.25 & 68.66 \\
\hline 32 & 49.70 & 60.50 & 73.64 & 43.99 & 55.67 & 70.45 \\
\hline 33 & 51.50 & 62.68 & 76.30 & 46.50 & 58.85 & 74.49 \\
\hline 34 & 53.24 & 64.81 & 78.90 & 47.33 & 59.91 & 75.83 \\
\hline 35 & 54.95 & 66.89 & 81.42 & 49.52 & 62.68 & 79.35 \\
\hline 36 & 56.61 & 68.91 & 83.88 & 51.18 & 64.79 & 82.02 \\
\hline 37 & 58.22 & 70.87 & 86.27 & 52.70 & 66.72 & 84.46 \\
\hline 38 & 59.79 & 72.79 & 88.61 & 54.18 & 68.60 & 86.85 \\
\hline 39 & 61.32 & 74.65 & 90.87 & 55.01 & 69.65 & 88.18 \\
\hline 40 & 62.82 & 76.47 & 93.09 & 56.43 & 71.46 & 90.48 \\
\hline & & & & \\
\hline
\end{tabular}

Fuente: Base de datos del servicio de medicina fetal del Instituto Nacional Materno Perinatal 2011-2015.

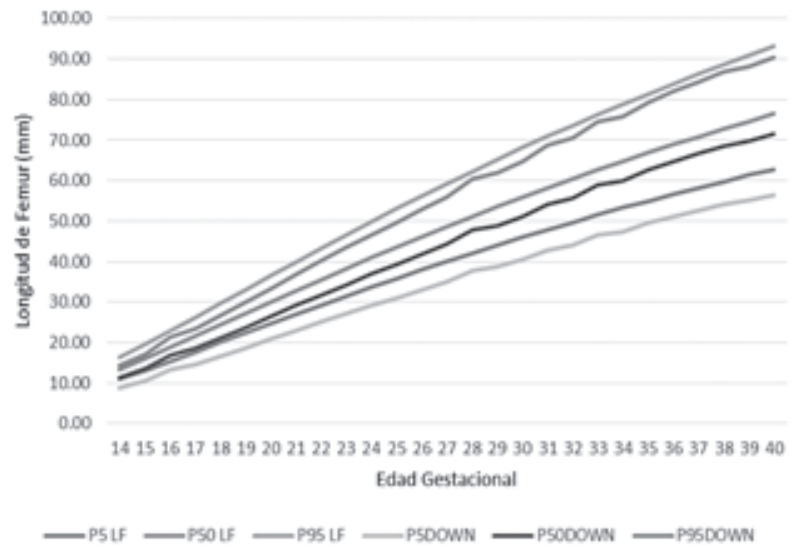

Gráfico 3. Comparacion de la curva de longitud de femur de los fetos sanos con los fetos Sindrome de Down del Instituto Nacional Materno Perinatal 20112015

Fuente. Base de datos del servicio de medicina fetal del Instituto Nacional Materno Perinatal 2011-2015.

Interpretación. En la tabla 3 y gráfico 3 se puede observar que al comparar los fetos sanos y los fetos con síndrome de Down, los valores de longitud del fémur son claramente diferentes en el síndrome de Down, con una tendencia a valores bajos, los cuales varían entre 8,78 mm el mínimo y el máximo con $56,43 \mathrm{~mm}$ ubicándose en el percentil 5, para el percentil 50 fue 11,18 a $71,46 \mathrm{~mm}$ y para el percentil 90 fue de 14,23 a $90,48 \mathrm{~mm}$. Por lo tanto en todas las edades gestacionales la curva para longitud del fémur de los fetos con síndrome de Down se encontró por debajo de la curva de longitud del fémur en fetos sanos.

Tabla 4. Estimacion de riesgo e intervalo de confianza de la longitud del femur de fetos sanos y fetos con Sindrome de Down a las 20-26 semanas.

\begin{tabular}{lccr}
\hline & Estimación de riesgo & \\
\hline & & \multicolumn{2}{c}{ Intervalo de confianza de } \\
& Valor & \multicolumn{2}{c}{$\mathbf{9 5}$} \\
\cline { 3 - 4 } & & Inferior & Superior \\
\hline $\begin{array}{l}\text { Odds ratio para FL31 } \\
(1,00 / 2,00)\end{array}$ & 2,541 & 1,071 & 6,650 \\
$\mathrm{~N}$ de casos válidos & 1146 & & \\
\hline
\end{tabular}

Fuente: Base de datos del servicio de medicina fetal del Instituto Nacional Materno Perinatal 2011-2015.

Interpretación. La posibilidad de que sea un feto con síndrome de Down es 2,5 veces más probable cuando la longitud del fémur es $<=31 \mathrm{~mm}$ entra las semanas 20 a 26 de gestación y cuyo valor fluctúa entre 1,071 $-6,650$. 


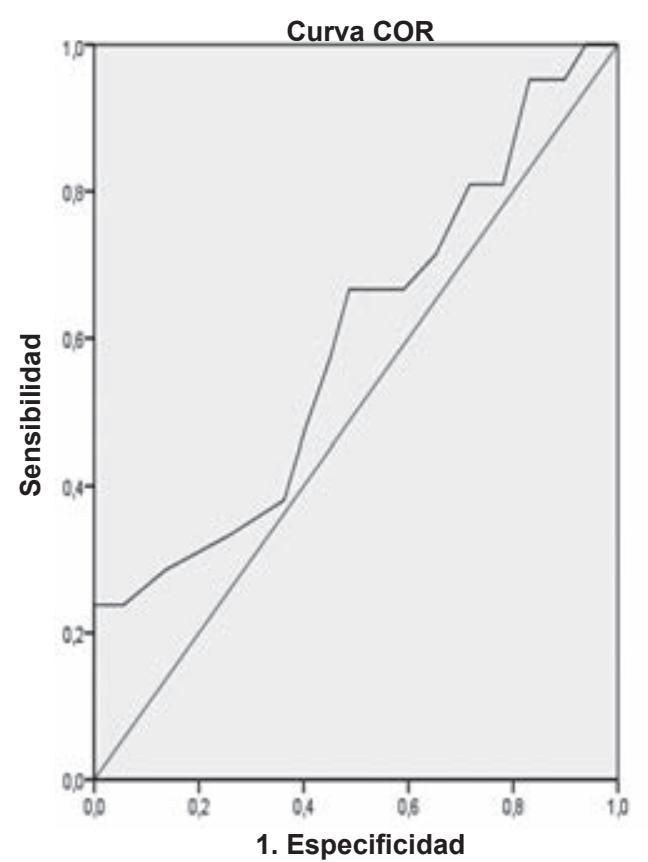

Los segmentos de diagonal se generan mediante empates.

Gráfico 4. Curva y coordenadas COR para sensibilidad y especificidad de longitud del femur a las $20-26$ semanas de gestación.

Fuente: Base de datos del servicio de medicina fetal del Instituto Nacional Materno Perinatal 2011-2015.

Tabla 5. Coordenadas de la curva Variable(s) de resultado de prueba: Longitud

\begin{tabular}{ccc}
\hline Positivo si es menor que $^{\mathrm{a}}$ & Sensibilidad & 1 - Especificidad \\
\hline 22,0000 &, 000 &, 000 \\
23,5000 &, 048 &, 000 \\
25,0000 &, 095 &, 000 \\
26,5000 &, 143 &, 000 \\
28,0000 &, 238 &, 000 \\
29,0500 &, 238 &, 015 \\
29,5500 &, 238 &, 016 \\
30,5000 &, 238 &, 056 \\
31,5000 &, 286 &, 136 \\
32,5000 &, 333 &, 259 \\
33,5000 &, 381 &, 363 \\
34,5000 &, 476 &, 403 \\
35,5000 &, 571 &, 450 \\
36,5000 &, 667 &, 487 \\
37,5000 &, 667 &, 540 \\
38,5000 &, 667 &, 591 \\
39,5000 &, 714 &, 652 \\
40,5000 &, 810 &, 717 \\
41,5000 &, 810 &, 780 \\
42,5000 &, 952 &, 831 \\
43,5000 &, 952 &, 898 \\
44,5000 & 1,000 &, 939 \\
45,5000 & 1,000 &, 983 \\
47,0000 & 1,000 & 1,000 \\
\hline
\end{tabular}

Fuente: Base de datos del servicio de medicina fetal del Instituto Nacional Materno Perinatal 2011-2015.
Interpretación. Se observó que hay una sensibilidad de un $23 \%$ con una tasa de falsos positivos de $0 \%$, es decir no hay tasa de falsos positivos, pero la sensibilidad de longitud de fémur entre las 20-26 semanas de gestación es baja, entonces la sensibilidad para detectar síndrome de Down es baja, pero ningún sano está dentro.

\section{Contrastación de hipótesis}

\section{Pruebas de chi-cuadrado}

\begin{tabular}{lccc}
\hline & Valor & $\begin{array}{c}\text { Grado de } \\
\text { libertad }\end{array}$ & $\begin{array}{c}\text { Sig. asintótica } \\
\text { (bilateral) }\end{array}$ \\
\hline Chi-cuadrado de Pearson & $759,219^{a}$ & 107 &, 000 \\
N de casos válidos & 3623 & & \\
\hline
\end{tabular}

Fuente: Base de datos del servicio de medicina fetal del Instituto Nacional Materno Perinatal 2011-2015.

Hi: Existen diferencias entre las longitudes de fémur de fetos sanos del INMP respecto a fetos con síndrome de Down del INMP.

HO: No hay diferencia entre las longitudes de fémur de fetos peruanos sanos del INMP respecto a fetos con síndrome de Down del INMP.

Se encontró en el estudio que el valor de la prueba de Chi Cuadrado es 759,219 y el valor de significancia 0,000 (menor a 0,05 ) se encontró evidencia estadísticamente significativa para rechazar la hipótesis nula y aceptar la hipótesis alterna, es decir que existen diferencias entre la longitud de fémur de los fetos con síndrome de Down comparados a la longitud de fémur de los fetos sanos del Instituto Nacional Materno Perinatal, $2011-2015$.

Gráfico 5. El femur corto no es un buen marcador de Sindrome de Down en fetos atendidos en el Instituto Nacional Materno Perinatal periodo 2011-2015

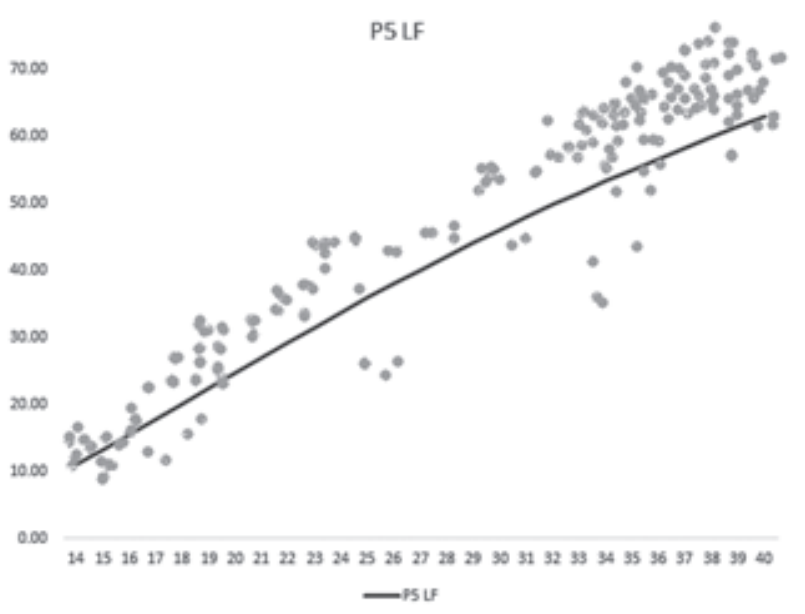

\section{DISCUSIÓN}

En los resultados obtenidos en este estudio sobre la comparación de las curvas de la longitud de fémur de los fetos sanos con respecto a los fetos con síndrome de Down, se encontró que en el percentil 95 para los fetos sanos las medidas fluctuaron entre 16,34 a $93,09 \mathrm{~mm}$ y para los fetos con síndrome de Down entre 14,23 a $90,48 \mathrm{~mm}$, Asimismo se encontró en el percentil 50 con 13,42 a $76,47 \mathrm{~mm}$ para fetos sanos y de 11,18 a $71,46 \mathrm{~mm}$ para fetos con síndrome de Down, de igual manera para el percentil 5 se encontró medidas de 11,03 a $62,82 \mathrm{~mm}$ 
para fetos sanos y de $8.78 \mathrm{~mm}$ a $56.43 \mathrm{~mm}$ para fetos con síndrome de Down, encontrándose que las curvas para el percentil 5, 50 y 95 de los fetos con síndrome de Down, se encuentran por debajo de la curva de la longitud del fémur de los fetos sanos. Este resultado se asemeja con el de Berstein $\mathrm{S}$ y col ${ }^{11}$, cuyo valores de longitud de fémur oscila entre 20 a $80 \mathrm{~mm}$ aproximadamente y la edad gestacional comprendida entre 15 a 39 semanas, en el cual menciona que encontró diferencias entre las curvas de longitud de fémur para fetos sanos y fetos con síndrome de Down, hallando que la curva de fetos con síndrome de Down se encuentra por debajo respecto a la curva de la población sana. Este resultado se asemeja con el de Longo D, De Figueiredo D, Cicero S, Sacchini C and Nicolaides NK ${ }^{27}$ en donde midió la longitud del fémur y humero desde las 11 a las 14 semanas de gestación, hallando que las medidas de longitud del fémur en los fetos con síndrome de Down, para las semanas 11-14 estaban por debajo del percentil 5 y 95 de la normalidad. Así mismo este resultado concuerda con el estudio de Nyberg $A$ y col${ }^{8}$ en donde señala que los fetos con síndrome de Down son más propensos a demostrar medidas de longitud de fémur por debajo y más cortos comparados con los fetos normales donde también señala que el valor predictivo de la longitud del fémur para síndrome de Down es mucho más bajo que originalmente se sugirió en otras literaturas. Así mismo en los resultados obtenidos se observó que hay una sensibilidad de un $23 \%$ con una tasa de falsos positivos de $0 \%$, resultados que difieren con Borgida A, Zelop C, De Roche M, Bolnick A, Egan $\mathrm{J}^{6}$ en donde señalan que fémur corto al tiene una sensibilidad del $24 \%$ con una tasa de falsos positivos del $4,7 \%$.

Financiamiento: autofinanciado.

Conflicto de interés: Los autores declaran no tener algún conflicto de interés.

\section{REFERENCIAS BIBLIOGRÁFICAS}

1. Bermejo E, Cuevas L, Martínez M. Informe anual del ECEMC sobre vigilancia epidemiológica de anomalías congénitas en España: Datos del período 1980-2010. Rev Dismor Epidemiol. 2011;6(1): 84-121.

2. Jané M, Prats R, Plasencia A et al. Protocolo de diagnóstico prenatal de anomalías congénitas fetales, Generalitat de Catalunya Departamento de Salud; 43:15-16.

3. Instituto Nacional Estadística e Informática. Boletín estadístico 2014. Lima - Perú. 2014:1-36.

4. Waitzman N, Romano P, Scheffler R. Economic costs of congenital anomalies. Morb Mortal Wkly Rep. 1995: 44:37.

5. Breathnach FM, Fleming ANN, Malone FD. The Second Trimester Genetic Sonogram. Am J Med Genet part C (Seminars Med Genet). 2007; 145:62-72.

6. Borgida L, Zelop C, DeRoche M, Bolnick A, Egan J. Down syndrome screening using race-specific femur length. Mosby 2003; 979:977-979.

7. Lorente R. Revisión sistemática y metaanálisis del rendimiento diagnóstico de los hallazgos ecográficos componentes del sonograma genético realizado en el segundo trimestre de gestación para la detección de Síndrome de Down. tesis doctoral. Madrid, España. Universidad Complutense de Madrid, 2016:276 pp.

8. Nyberg et al. Femur length shortening in the detection of Down syndrome: Is prenatal screening feasible? Am J Obstetra Gynecol 1990; 6:1247-1252.

9. Weisz et al. The Association of Midtrimester Short Femur with Perinatal Outcome. J Ajog 2006; 62: 229-235.

10. Parra $M$ et al. screening for trisomy 21 during the routine second-trimester ultrasound examination in an unselected Chilean population. Ultrasound Obstet Gynecol 2007; 30: 946-951.

11. Bernstein S, Saller D, Catov J, Canavan T. Ultrasonography estimates of fetal growth in fetuses affected by trisomy 21 . IJGO. 2016; 4:1-4.

12. Cuartas A. Marcadores ecográficos de aneuploidías del primer y del segundo trimestre del embarazo. XVI Curso de Actualización en Ginecología y Obstetricia; 2008:11-26.

13. Ventura $W$ et al. Resultados perinatales en fetos del segundo trimestre con fémur cortó aislado. Rev Per Ginecol Obstet. 2011; 57: 33-37.

14. Kovac A et al. Maternal Ethnicity and Variation of Fetal Femur Length Calculations When Screening for Down Syndrome. J Ultrasound Med 2002; 21:719-722.

15. Todros $T$ et al. Fetal short femur length in the second trimester and the outcome of pregnancy. BJOG January 2004; 111:83-85.

16. Mathiesen J, Aksglaede L, Skibsted L, Petersen O,Tabor A. Outcome of fetuses with short femur length detected at second-trimester anomaly scan: a national survey. Ultrasound Obstet Gynecol 2014; 44: 160-165.

17. Villamonte W, Jerí M, De la Torre C. Biometría fetal e Índice de líquido amniótico de 14 a 41 semanas a 3400 msnm y su comparación con tablas de otros niveles de altura fetal. Acta Med Per. 2013; 30:1

18. Johnson $M$ et al. Combining Humerus and femur length for improved Ultrasonographic identification of pregnancies at increased risk for trisomy 21. J Obstet Gynecol. 1995; 1235:1229-1235.

19. Nyberg DA, Souter VL. Use of genetic sonography for adjusting the risk for fetal Down syndrome. Semin Perinatol. 2003; 27(2):130-44.

20. Harper LM, Gray D, Dicke J, Stamilio DM, Macones GA, Odibo AO. Do Race-Specific Definitions of Short Long Bones Improve the Detection. J Ultrasound Med. 2010;29:231-235.

21. Borgida A et al. Influence of fetal gender on femur length when screening for Down Syndrome. University of Connecticut 2003; 15: 150-165.

22. Oliva J . Ultrasonografía Diagnóstica fetal, obstétrica y Ginecología, primera edición. La Habana, Editorial Ciencias Médicas. 2010:45-50.

23. Pérez A . Síndrome de Down. Revista de Actualización Clínica. 2014; 45(5): 2357-2361.

24. Grandi C, López F. Estimación de la edad gestacional: Revisión de la Literatura. Rev. Del Hospital Materno Infantil Ramón Sardá. Buenos Aires, Argentina.2004;23 (3):138-143.

25. Taboada G , Lafuente E, Chino J, Rada A, Cuti M. Síndrome de Down y edad materna (Tesis de Maestría). Bolivia: Universidad Mayor de San Andrés, 2010:141 pp.

26. Hernández R, Fernández C, Baptista M. Metodología de la Investigación. 5 ed. MC Graw Hill Companies México; 2010:80pp

27. Longo A, Figueredo D, Cicero S, Sacchini C, Nicolaides K. Femur and Humerus length in trisomy 21 fetuses at $11-14$ weeks of gestation. ISUOG. 2014; 23: 143-147. 
28. Flores J, Ruiz E. Síndrome de Down. Rev. Síndrome Down. 2003;17(3): 48-53.

29. Cunningham, Leveno, Bloom, Hauth, Rouse, Spong. Williams de Obstetricia. 23 era edición. Estados Unidos de Norte América. McGraw-Hill Interamericana Editores; 2011: P. 266-287.

30. A Basavilvazo, et al. Diagnóstico Prenatal del Síndrome de Down. México. CENETEC; 2011. P.p 7-8.

31. Rao R, Platt L. Ultrasound screening: Status of markers and efficacy of screening for structural abnormalities. Elsevier Inc. 2015; 11: 3-11.

32. Kagan KO, Valencia C, Livanos $P$, Wright D, Nicolaides $\mathrm{KH}$. Tricuspid regurgitation in screening for trisomies 21, 18 and 13 and Turner syndrome at $11 \mathrm{p} 0$ to $13 \mathrm{p} 6$ weeks of gestation. Ultrasound Obstet Gynecol. 2009; 33(1):18-22.

33. Prefumo F, Sethna F, Sairam S, Bhide A, Thilaganathan B. First-trimester ductus venosus, nasal bones, and Down syndrome in a high-risk population. Obstet Gynecol. 2005; 105(6): 1348-1354.
34. Nyberg A, Souter L, El-Bastawissi A, Young S, Luthhardt F, Luthy A. Isolated sonographic markers for detection of fetal Down syndrome in the second trimester of pregnancy. $J$ Ultrasound Med. 2001; 20(10):1053-1063.

35. Coco C, Jeanty P. Isolated fetal pyelectasis and chromosomal abnormalities. Am J Obstet Gynecol. 2005; 193:732-738

36. Nyberg A et al. Humerus and femur length shortening in the detection of Down's syndrome. Am. J Obstet Gynecol 1993; 168: 534-8.

37. Ramírez J. Anomalías Fetales. Temas Obstetricia 2010; 11 : $1-2$.

\section{Correspondencia:}

Dirección: Av. Javier Prado Este 175, Piso 3. Of. 304 -San IsidroLima.

Teléfono: (51 1) 447-8056 Celular: (51 1) 984-840625

E-mail: antoniolimay@hotmail.com

\section{ORCID iDs}

Antonio Limay Rios

Jaime Ingar Pinedo

Walter Castillo Urquiaga

Walter Ventura Laveriano (i) https://orcid.org/0000-0001-6012-3705

(di) https://orcid.org/0000-0001-6472-7899

(6i) https://orcid.org/0000-0002-1054-7398

(d) $\mathrm{https://orcid.org/0000-0002-2888-1394}$ 